\title{
Optimal calibration of directional velocity probes
}

\author{
Stephen C. McParlin ${ }^{1}$, Samantha. S. Ward ${ }^{2}$ and David M. Birch. ${ }^{3}$ \\ University of Surrey, Guildford, Surrey, GU2 7XH, UK
}

\begin{abstract}
A novel approach has been considered for the formal process of calibrating multiple hole pressure probes for use in wind tunnels. Rather than determining the attitude angles of a probe and subsequently flow angularity for a fixed probe, either by linear interpolation between sample points or through the use of piecewise functional fits, the outputs from the probe are mapped as continuous functions across the angular test space, using a set of sample points derived from Optimal Design of Experiments. This offers the potential of more accurate probe calibrations across a wider range of flow onset angles, with fewer sample points than currently used for the same purpose. Proof-of-concept tests using a fivehole probe have indicated that the approach is viable, while examination of fits to legacy data from prior tests indicates that the approach is easily extendable to probes with an arbitrary number of holes, and to multiple hot-wire installations.
\end{abstract}

\section{Nomenclature}

$\begin{array}{ll}\text { ANOVA } & =\text { Analysis of Variances } \\ \text { DoE } & =\text { Design of Experiments } \\ \mathbf{i}, \mathbf{j}, \mathbf{k} & =\text { Orthogonal quaternion vectors, where } \mathbf{i}^{2}=\mathbf{j}^{2}=\mathbf{k}^{2}=\mathbf{i j k} \mathbf{k}=-1 \\ \mathrm{P}_{\mathrm{i}} & =\text { Measured pressure at point } \mathbf{i} \\ \mathbf{q} & =\text { Unit quaternion }\left(\mathbf{q}=q_{1}+q_{2} \mathbf{i}+q_{3} \mathbf{j}+q_{4} \mathbf{k}\right) \\ \mathrm{C}_{\mathbf{P}} & =\text { Pressure coefficient } \\ \mathrm{C}_{\alpha} & =\text { Differential pressure function with pitch at low coning angle } \\ \mathrm{C}_{\beta} & =\text { Differential pressure function with yaw at low coning angle } \\ \mathrm{C}_{\theta} & =\text { Differential pressure function with pitch at high coning angle } \\ \mathrm{C}_{\varphi} & =\text { Differential pressure function with yaw at high coning angle } \\ \mathrm{RSM} & =\text { Response Surface Model } \\ \alpha & =\text { Angle of incidence } \\ \beta & =\text { Angle of sideslip } \\ \phi & =\text { Euler angle in roll } \\ \theta & =\text { Euler angle in pitch } \\ \psi & =\text { Euler angle in yaw } \\ \sigma & =\text { Standard deviation }\end{array}$

\section{Introduction}

$\mathrm{T}$

HE calibration of multi-hole pressure probes has been an issue of some interest, owing to the large amount of facility time typically required to obtain calibration data sets of sufficient density and quality to yield high confidence experimental results. Though the behaviour of an ideal multi-hole pressure probe may be obtained analytically ${ }^{1}$, even small manufacturing errors render an experimental calibration necessary. Though there has been some effort toward applying advanced numerical techniques and neural-network algorithms toward probe calibration $^{2}$, the most commonly used calibration methods are least-squares or piecewise-polynomial functional approximations ${ }^{3,4}$, though direct linear interpolation schemes are also frequently encountered ${ }^{5,6}$. For both techniques, ${ }^{1}$ Laboratory Assistant, Division of Mechanical, Medical and Aerospace Engineering, Associate Fellow AIAA.

${ }^{2}$ Doctoral Candidate, Division of Mechanical, Medical and Aerospace Engineering.

${ }^{3}$ Lecturer in Aerospace Engineering, Division of Mechanical, Medical and Aerospace Engineering. 
the quality of the resultant data increases with the number of calibration points. Significantly, Sumner ${ }^{7}$ showed that for flows of large angularity, the direct interpolation method resulted in lower uncertainties, while the functional approximation method was more accurate for flows of small angularity. Consequently, neither technique can be optimal for flows spanning both large and small angles.

In nearly all cases, either rectangular or logarithmic calibration grids are used, primarily as a matter of convenience; indeed, irregularly-spaced calibration points render some calibration schemes impractical. However, the sensitivity of the sensors is not uniform throughout the calibration space, so a grid of arbitrary spacing is also necessarily suboptimal.

This paper presents a novel approach to the calibration of multi-hole pressure probes, derived from the theory of optimal design of experiments (DoE) and extends this technique to the calibration of multi-sensor hot-wire probes. Optimisation techniques are used to derive a set of input values in an arbitrary set of dimensions, in both space and other variables for calibration, which are then sampled, returning multiple responses. Measured values for the sample set of points are then fitted as response surfaces, using formal statistical methods (ANOVA) to determine the type of fit, eliminate insignificant terms in the relationships used to fit the data, identify outliers and determine the bounds of validity for each of the relationships obtained.

The calibration of experimental measurement equipment is fundamental to the accuracy and repeatability of all quantitative observations. There are numerous factors involved, not least the time taken for measurements supporting calibration. This is particularly important for instruments such as hot-wires, which are subject to time and temperature-dependent drift and therefore require frequent recalibration. Formalized approaches to calibration (which can be automated) reduce the amount of time required and ensure that repeatability between calibrations can be assessed rapidly. This is important for both the formal and technical quality assurance associated with subsequent data measurements.

Introducing an optimal DoE-based approach assures repeatability between tests by ensuring that a standard set of calibration points are used that offer minimum probability of error while limiting the number of sample points to the minimum required to achieve accurate, continuous fits to the measurements. Representation of the measured variables as a continuum also allows rapid inversion of the calibration matrix for subsequent experimental observations.

\section{Calibration of multi-hole pressure probes}

\section{A.Current practice}

\section{Five hole probes}

The principle behind the calibration of five-hole probes is formally similar to that for seven-hole probes, and is described in detail by Treaster and Yocum ${ }^{8}$; the only difference is that the coefficients are obtained as the differences between pressures obtained at single holes rather than as averages over multiple holes (the most obvious example of this is perhaps the numerator of Eq. 1). As a consequence, five-hole probes tend to have a smaller range of sensitivity than seven-hole probes, though techniques have been presented for extending their range 9 .

2.Seven-hole probe layout and numbering convention

The function of a seven-hole probe is to derive local flow velocity components from pressure measurements. The layout of the probe differs from that of a five-hole probe by having a hexagonal, rather than cruciform, arrangement of holes; these are arranged in close-packed configuration around the central hole, as shown in figure 1 . The holes are conventionally numbered clockwise from 1-7, with 1 being at the 6 o'clock position and the central hole numbered as 7 .

In normal use, the probe will be orientated parallel to the free stream direction. Hence, when aligned parallel to an undisturbed free-stream, the pressure coefficient measured at the central hole, $\mathrm{C}_{\mathrm{P} 7}$ :

$$
C_{P 7}=\frac{P_{7}-P_{\text {static }}}{P_{\text {total }}-P_{\text {static }}} \approx 1.0
$$

while, depending on probe head geometry, the remaining pressure holes should record values of pressure coefficient somewhere between stagnation and free-stream static pressure:

$$
C_{P 1} \approx C_{P 2} \approx C_{P 3} \approx C_{P 4} \approx C_{P 5} \approx C_{P 6} \geqslant 0.0
$$

Hence, for a perfectly aligned probe with radial holes parallel to the onset flow the results are similar to those for a Pitot-static tube. Indeed, for small angles, where the peak pressure is recorded at hole 7, the mean pressure from holes 1-6 is taken as a measure of local static pressure, while the pressure measured at hole 7 is taken as local total pressure, giving a measure of mean local dynamic pressure and hence flow speed. 


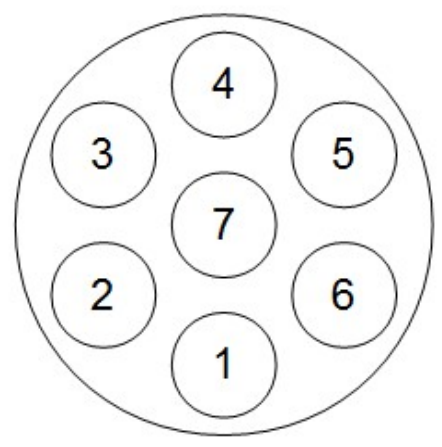

Fig. 1 Radial arrangement of pressure holes on a seven-hole probe, looking aft

However, as shown in Fig.2, the radial pressure holes are orientated towards the free-stream direction, and thus the pressure measured by these will be above local static pressure.

3.Treatment at low coning angle

The local flow angle is determined from differential pressures between the radial holes. At low angles of pitch, $\theta$, where maximum pressure is at hole 7, the differential pressures between holes 1 and 4 are used to derive a function, $\mathrm{C}_{\alpha}$, while the difference between the mean of pressures at holes 2 and 3 and the mean of pressures at holes 5 and 6 are used to derive a function $\mathrm{C}_{\beta}$, i.e.

$$
C_{\alpha} \equiv \frac{P_{4}-P_{1}}{P_{7}-P_{\text {mean }}}
$$

and

$$
C_{\beta} \equiv \frac{\frac{1}{2}\left(P_{5}+P_{6}\right)-\frac{1}{2}\left(P_{3}+P_{2}\right)}{P_{7}-P_{\text {mean }}}
$$

Although given subscripts more proper to angles of incidence and sideslip respectively, these are both functions of the rotational degrees of freedom in pitch and yaw. Hence the calibration process for these functions involves traversing the probe through $\theta$ and $\psi$ for the space in which $\mathrm{P}_{7}$ is the maximum pressure recorded by the probe head. However, the boundary in $\theta$ at which the maximum pressure shifts from hole 7 is not known a priori and indeed, may be a function of Reynolds number, with implications for the extent of the validity of any calibration.

For the maximum pressure at $\mathrm{P}_{7}$, then,

$$
C_{\alpha}=f_{1}(\theta, \psi)
$$

and

$$
C_{\beta}=f_{2}(\theta, \psi)
$$

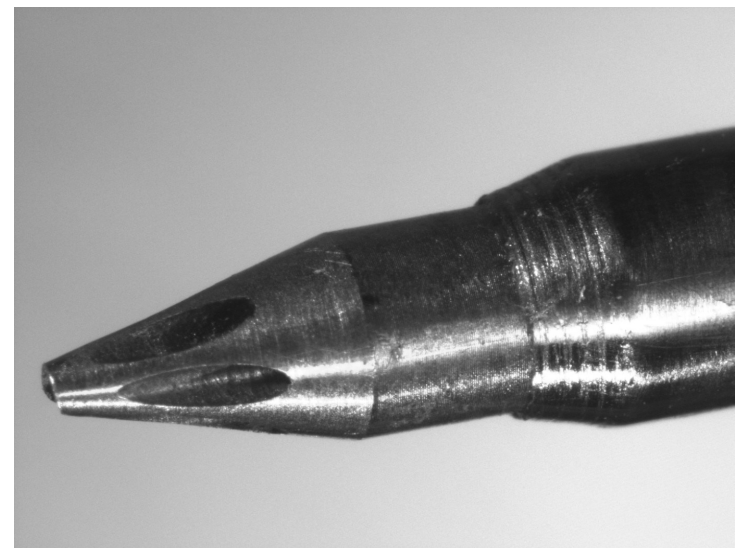

Fig. 2 Head of miniature 7-hole probe

and

$$
C_{i, \phi} \equiv \frac{P_{c w}-P_{a c w}}{P_{i}-P_{\text {mean }}}
$$

Then, when the maximum pressure is not at $\mathrm{P}_{7}$ :

$$
C_{i, \theta}=f_{i, 1}(\theta, \phi)
$$

and

$$
C_{i, \phi}=f_{i, 2}(\theta, \phi)
$$

Equations (2) and (3), together with (7) and (8), yield seven different sets of fitted representations in matrix form, which need to be inverted to return the angle of the flow relative to the probe when in normal use. 


\section{Summary}

It should be noted that:

1)The logic of deciding which process to use is determined solely by the location of the maximum pressure measurement.

2)There are effectively seven independent calibrations involved, one for each of the holes at the probe tip, corresponding to non-overlapping regions of the rotational vector of the probe.

These observations have a number of implications:

1)There is no continuity, either explicitly or implicitly, between the different calibrated regions.

2)In the case where the maximum pressure is measured as equal at two adjacent points, the logic fails. No account is taken of measurement accuracy, or whether the confidence intervals for these overlap.

3)There is no guarantee that the local stagnation pressure is registered at any of the holes, particularly as the peak pressure moves from one region to another.

4)There is no guarantee that the true local static pressure is registered at any of the holes, or that the mean values, as currently defined, are true representations of the actual local static pressure.

5)As the effective flow onset angle increases, there are no specific checks as to which of the pressure holes is producing valid results. In particular, the onset of flow separation at the central hole, which should be a limiting factor on maximum useable angle, is not determined or used.

\section{B. Suggested areas for improvement}

It should be noted that, although the relations obtained for $\mathrm{C}_{\alpha}, \mathrm{C}_{\beta}, \mathrm{C}_{\theta}$ and $\mathrm{C}_{\varphi}$ are derived using the absolute measured pressures, the results will be identical if $\mathrm{P}_{1}-\mathrm{P}_{7}$ are described as incremental pressures relative to freestream static pressure, or indeed, pressure coefficients, normalised by free-stream dynamic pressure. Using the last of these approaches, it is possible to apply a check for validity of individual pressure measurements. If the flow is assumed to be adiabatic, then the stagnation pressure seen by the head of the probe will always be less than freestream total pressure. This provides a maximum value of pressure coefficient beyond which measurements are suspect. Conversely, the minimum pressure coefficient experienced by any pressure hole under attached flow conditions can be determined by inspection of the data measured during the calibration process. Hence, rather than determining the validity of pressure measurements on the leeward side of the probe head based on measurements at the windward side, it should be possible to determine the validity of an individual point based on whether it falls within prescribed bounds. The same criterion used to determine the validity of the pressure measured at the most leeward point can also be applied, at higher $\theta$, to determine the validity of the central point, and hence the limit of applicability of the probe.

As the logic defining the function of the probes breaks down at the boundaries of the calibration spaces of adjacent holes, where pressure measurements at the holes become equal, it is desirable to find an alternative approach in which these boundary regions overlap, so as to provide continuity and potentially increased accuracy. Rather than following a logic dictated by the location of the maximum pressure, it should be possible to map the spaces where individual pressure measurements are valid, and then create a Venn diagram of overlapping maps, allowing all of the available and valid measurements to be used to determine the flow onset angle. Hence, at the centre of the Venn diagram, all seven pressures should be valid, while at the boundaries of the Venn diagram, only the pressure at the point closest to windward will be valid. Between these extremes, the number of overlapping measurements will reduce progressively, giving a gradual reduction in available accuracy as the onset flow angle becomes more extreme. The current scheme assumes that the data for the three holes surrounding the most windward point remain attached up to the limit of coning angle for the probe.

At the point of investigating the feasibility of alternative approaches, a number of issues needed to be resolved:

1)The criteria to be used to determine the validity of pressure measurements at a given hole.

2)How to delineate the boundaries of validity for each pressure hole in terms of probe attitude.

3)How to merge angular position information from overlapping data sets.

4)Whether alternative variants of the differential pressure functions, using all valid data at a given probe attitude, can be used.

To enable an alternative approach, it is necessary to characterize the relationship between pressure coefficient and probe attitude over the operating range of the probe. This can be achieved through the formal process of DoE, which is designed to minimize error in fitting relationships between input and output variables. 


\section{Optimal Design of Experiments}

\section{A. Design of Experiments}

The field of design of experiments seeks to produce formal processes and schemes for quantitatively determining the sensitivity of outputs to variations in associated inputs. In practice, this means designing efficient sampling schemes to identify which factors are significant in the generation of an output, and then characterizing the relationships between input factors and output responses. In classical design of experiments, experimental design schemes start with full-factorial schemes, in which sample points are chosen at the centre, vertices and face centres of hypercubes of the dimensions of the input factors, and then alternative schemes with progressively smaller sample sets are used. For a 2D hypercube, a full-factorial sample set to support a quadratic fit would consist of nine sample points arranged as a regular $3 \times 3$ array. For a $3 \mathrm{D}$ hypercube, the corresponding number of sample points in a fullfactorial scheme would be 27 , in a $3 \times 3 \times 3$ arrangement. As can be seen, the number of sample points in a quadratic full-factorial scheme varies as $3^{n}$, where $n$ is the number of factors involved.

The second element in Design of Experiments involves the analysis of sample data to formally determine the significance of individual or coupled factors. The analysis of variance (ANOVA) is a standard series of statistical tests used to evaluate the results of sampling, identify outliers and non-significant relationships and thus determine the relationship between input factors and output responses. The resulting relationships are then expressed in algebraic terms as a response surface model or RSM.

In optimal Design of Experiments, rather than follow a set sampling scheme, with the number of sample points being a consequence of the number of input factors, the objective is to optimize sampling locations given a fixed set of input variables and a fixed number of sample points. Among the advantages of this approach, the ability to make use of blocks of pre-existing sample points and cope with constraints on the boundaries of the sampling domain makes optimal DoE a great deal more flexible than classical schemes, while the rationale for the technique is to reduce the cost and time required to take samples.

\section{B. D-Optimality}

In the creation of an RSM for a function $y$, we are assuming that the domain of the model is continuous, such that it can be represented as a surface fit. For a simple polynomial fit $x=\left(x_{1}, \ldots, x_{m}\right)$ we can express $y$ as a polynomial with $p+1$ terms in $m$ variables.

$$
y=\sum_{i=0}^{p} \beta_{i} x_{1}^{\alpha_{11}} \ldots x_{m}^{\alpha_{m i}}+\epsilon
$$

where $\varepsilon$ is a small random error term which is assumed to be normally distributed with the variance, $\sigma^{2}$. This polynomial can also be expressed as:

$$
y=x_{p} \beta+\epsilon
$$

where

$$
\beta \equiv\left(\beta_{0, .} \ldots, \beta_{p}\right)^{T}
$$

isa vector of real coefficients which must be determined to fit the response surface model over the domain of interest.

Given $n$ data points $\left(X_{l 1}, \ldots X_{l m}\right), \ldots,\left(X_{n i}, \ldots X_{n m}\right)$, the $n$ values of $X_{p}$ for these $n$ data points can be arranged as a matrix $\mathbf{X}$ :

$$
X=\left[\begin{array}{ccc}
\left(X_{11}^{\alpha_{11}}, \ldots X_{1 \mathrm{~m}}^{\alpha_{1 \mathrm{~m}}}\right) & \ldots \ldots & ,\left(X_{11}^{\alpha_{1 \mathrm{p}}} \ldots X_{1 \mathrm{~m}}^{\alpha_{m p}}\right) \\
\cdot & \ldots \ldots . . & \cdot \\
\cdot & \ldots \ldots . & \cdot \\
\cdot & \ldots \ldots & \cdot \\
\left(X_{n 1}^{\alpha_{11}}, \ldots X_{n m}^{\alpha_{1 \mathrm{~m}}}\right) & \ldots \ldots & ,\left(X_{n 1}^{\alpha_{1 \mathrm{p}}}, \ldots X_{n m}^{\alpha_{m p}}\right)
\end{array}\right]
$$

For any such set of points, the value of $\beta$ resulting in the best possible fit is given by

$$
\left(X^{T} X\right)^{-1} X^{T} Y
$$

where

$$
Y \equiv\left(y_{i}, \ldots, y_{n}\right)^{T}
$$

is the vector of values of $y$ at the $n$ data points. In addition, the variance is given by 


$$
\beta_{\text {best }}=\left(X^{T} X\right)^{-1} \sigma^{2}
$$

D-optimality has the objective of finding the specific $n$ sample points which minimize this variance and hence providing an optimal estimate of $\beta_{\text {best }}$ by maximizing the determinant of $\mathbf{X}^{T} \mathbf{X}$. The sample size, $n$, to be used for Doptimality is a compromise between accuracy and the time or cost associated with the generation of samples.

The minimum value of $n$ required is that necessary to produce a non-singular matrix, which is $p$. In practice, the ratio of $n / p$ is chosen so as to give the minimum variance for a given cost or time. For a given value of $\mathrm{m}$, the value of $\mathrm{p}$ required to fit a quadratic polynomial then determines the number of sample points for a given ratio of $n / p$. For a quadratic description of a space with two independent variables, $p=6$. For a similar description of a space with three independent variables, $p=10$. As can be seen, the minimum sample set for a $\mathrm{D}$-optimal experimental design is smaller than for a full-factorial representation of the same space, while offering much larger savings in sample points for larger numbers of independent factors. For example, for a 7-dimensional space, the number of sample points for a quadratic full-factorial scheme is 2187 , while the minimum D-Optimal sample set for a quadratic RSM would be 120 sample points. Typically, however, the actual value of $n / p$ is determined by a compromise that gives an adequate level of fit to the sample data set, as determined by the regression coefficients and ANOVA analysis.

\section{Application of D-Optimality to probe calibration}

\section{A. Choice of attitude variables and bounds}

Although the differential force coefficients are conventionally written in terms of the translational angles, $\alpha$ and $\beta$, or the rotational Euler angles, $\theta, \psi$ and $\varphi$, the most relevant angles for the calibration and use of a probe are actually the coning angle relative to the wind axis and the circumferential angle of the probe about this axis. The probe attitude then has two degrees of freedom, although the probe itself is not put through any angle of roll. Angular transformations in general are non-commutative. Following best practice in flight mechanics and robotics, the angular space in $3 \mathrm{D}$ can be represented by a unit quaternion, where:

$$
\begin{gathered}
\vec{q}=q_{1}+q_{2} \vec{i}+q_{3} \vec{j}+q_{4} \vec{k} \\
|q|=1
\end{gathered}
$$

In the forward hemisphere of the probe, this maps to a definition where $q_{2}$ varies parametrically at some negative value while

$$
q_{3}^{2}+q_{4}^{2}=\text { constant }=\sqrt{1-q_{2}^{2}}
$$

is a constant. Note that unlike the Euler angle definition, this mapping can normally be extended into the rear hemisphere by allowing $q_{2}$ to assume a positive value. The limiting angle is determined by whether the results obtained from pressure measurement are valid and provide accurate information as to local flow angle at high angles. For a five-hole probe, current practice is that the probe is calibrated for angles of up to $\pm \pi / 4$ radians, while a seven-hole probe offers extended coning angle up to $\pm 3 \pi / 8$ radians. Because no real probes can be perfectly axisymmetric, the angle of roll about the wind axis should be $\pm \pi$ radians as well. No assumption is made about axisymmetric probe behaviour.

The bounds of the angular position variables, whether in Euler angles or unit quaternions, are fed into the wellestablished process for selection of D-optimal sample points. The resulting points are then converted back into pitch and yaw angles and used for calibration.

\section{B.Determining the form of the response surface models. \\ 1.Reviewing existing probe data}

To determine the pressure measurements from each hole of a probe, it was necessary to look at an existing data set, gathered as part of a conventional calibration process for a 7-hole probe. The facility used to produce these measurements was the 'A' tunnel in the Environmental Flow laboratory at the University of Surrey. This has an open return circuit and a working section of $900 \mathrm{~mm} \times 600 \mathrm{~mm}$. The tunnel speed was set at a nominal $10 \mathrm{~m} / \mathrm{s}$, and was actively controlled based on outputs from a local micromanometer driven by the tunnel Pitot-static system. The data was taken with respect to the hole array axis, ensuring that the pitch and yaw origin occurred at the angle for which the centre hole returned the maximum possible pressure. The error in probe position is estimated to be $\pm 0.2^{\circ}$.

The seven pressures from the probe were measured using an array of Honeywell 24PCEFA6D differential pressure sensors with Burr-Brown INA125 bridge driver/amplifiers, with reference pressures taken as the tunnel static. Based on manufacturer's specifications, the error in pressure is estimated to be $\pm 1 \%$. Both the wind speed control manometer and the pressure transducers are calibrated regularly. The probe results used were time-averaged 
over a period of 6 seconds at a sampling rate of $100 \mathrm{~Hz}$. The resulting time-averaged pressure measurement and associated variance for each data point was recorded during each run. However, for the purpose of determining the type of fit, the role of the data is qualitative, rather than quantitative. For production operation, however, quantitative accuracy and repeatability of pressure measurements is a significant factor in the accuracy of the method.
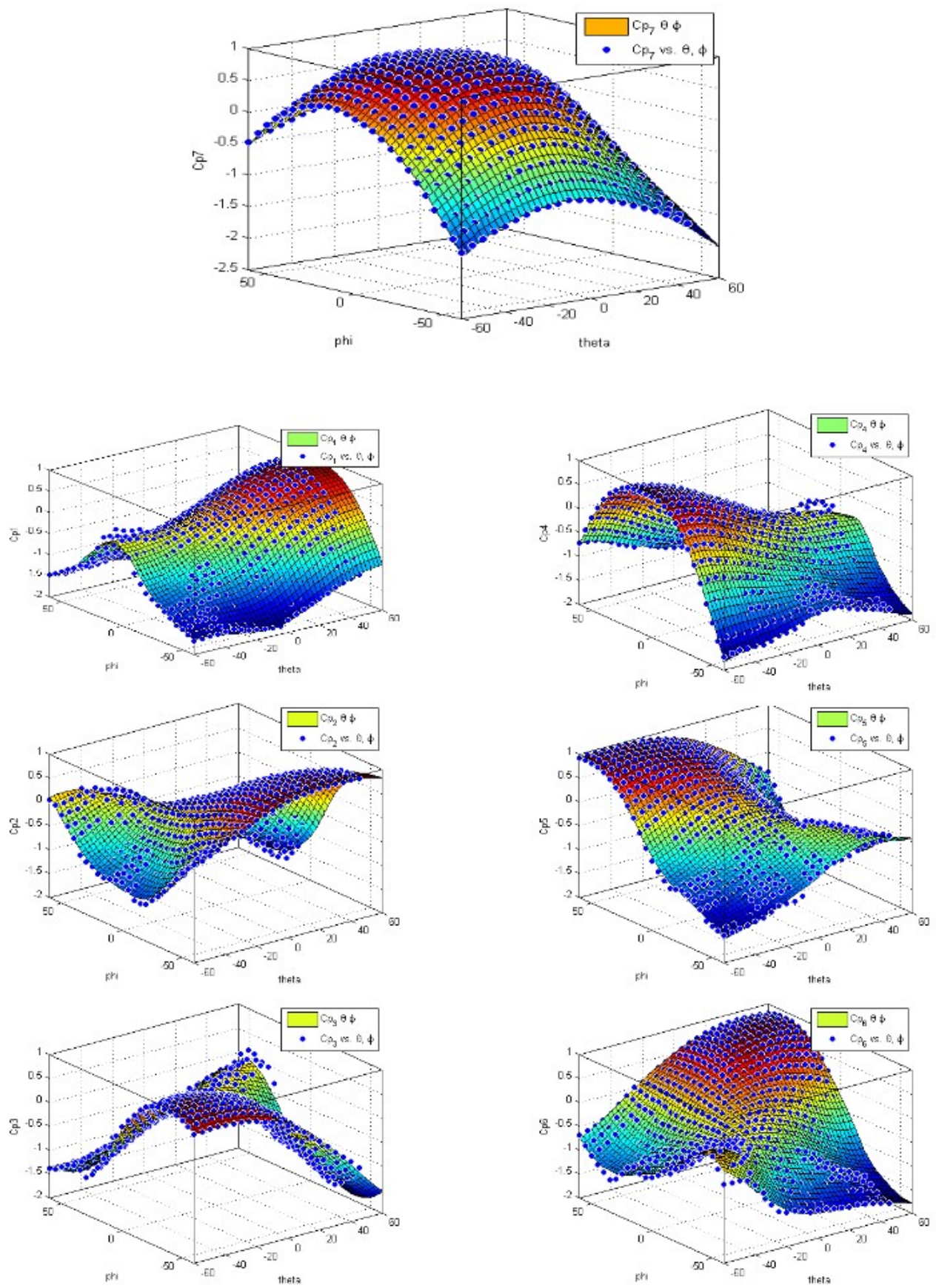

Figure 3 - Pressure measurements from a 7-hole probe

American Institute of Aeronautics and Astronautics 
The existing data had been generated using an automated process which controlled the probe attitude in pitch and yaw. Hence this data set was arranged in regular arrays of pressure at constant $\theta$ and $\psi$. comprising of approximately $25 \times 25$ points in each orientation. The corresponding outputs are shown in Figure 3 for each pressure hole. Note that the individual points shown are the time-averaged mean pressure, while the surfaces shown are piecewise quadratic. The top image in fig. 3 shows the variation of pressure with pitch and yaw for $\mathrm{C}_{\mathrm{P} 7}$, while those below on the left are for $\mathrm{C}_{\mathrm{P} 1}-\mathrm{C}_{\mathrm{P} 6}$ respectively. Those shown on the right are the diametrically opposite pressure ports to those on the left. While the results and surface fits are shown in the coordinate system in which they were measured, the effect of fitting these using cone and roll angle was also investigated. The surface fits to the existing data based on a polar representation appeared to be slightly worse in terms of regression coefficient, although it was not determined whether this was due to the data not being sampled as a regular array in these two variables.

2.Observations on existing pressure measurements

Examination of the pressure measurements, and the surfaces fitted through them, identifies some qualitative points:

1)The variation of pressure measurements with angle appeared reasonably regular. The fits to these surfaces are thus reasonably good, as measured in terms of regression coefficient, particularly in regions local to where the pressure is a maximum for a given hole. The variation for $\mathrm{C}_{\mathrm{P} 7}$ is regular throughout the range of angular calibration.

2)The accuracy of fit for the radial holes worsens away from the regions of maximum pressure, indicating that the fit of data could potentially be improved by eliminating some of the sample points furthest away from the maximum pressure measured for each hole, which is where the pressure measurements are most likely to be invalid.

3)Looking more closely at the location of maximum measured pressure, at the loci where the pressure at adjacent holes is equal and a maximum, neither port is registering the local total pressure, i.e. there is a maximum error in measurement of total pressure as maximum pressure moves from one port to the next. This emphasises the importance of having a continuous description of the mapping between total pressure and probe orientation.

3. Selection of sample points

The complexity of shape of the surfaces generated for the radial pressure ports suggested that it might be necessary to consider whether the use of a quadratic relationship was a limiting constraint on the process. The surfaces shown in fig. 3 were generated using a piecewise, rather than continuous, surfacing function, thus increasing the complexity of the task. In addition, while progressively increasing the number of sample points in a set, it was necessary to ensure that the number of repeat points in the sample set was controlled directly.

A commercial D-Optimal sample generation

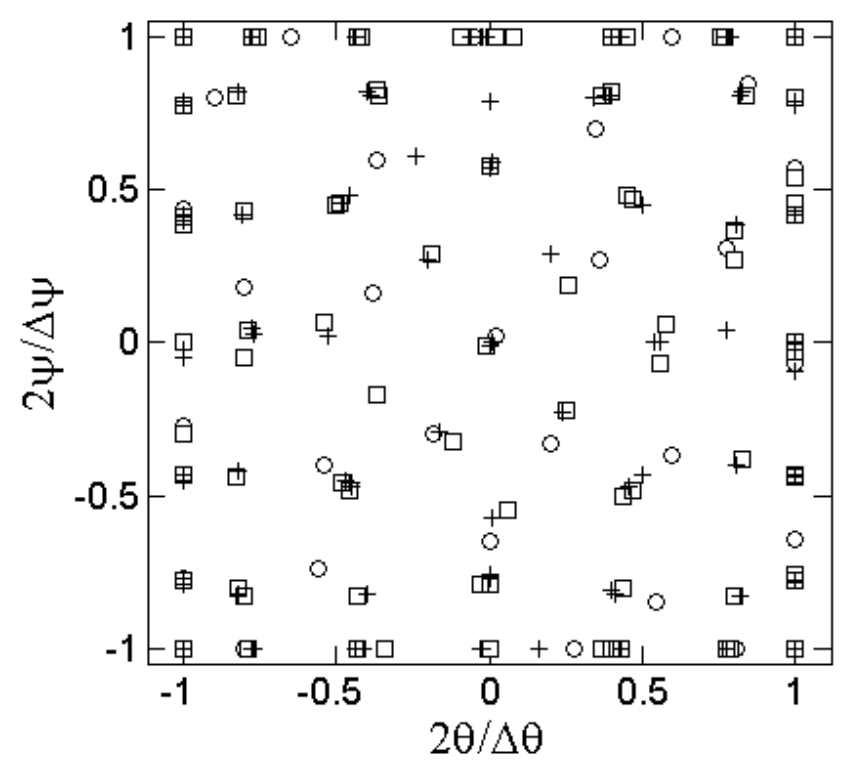

Fig 4. D-Optimal sampling sets of varying density software package was used to assess existing data from 5- and 7- hole probes and some triple-sensor hot wire calibrations. The likely goodness of fit for polynomial response surface models up to sixth order was assessed for the existing data. The predicted goodness of fit for the 7-hole probe increased with order of polynomial, with the sixth-order treatment being predicted to be marginally better than quartic or quintic treatments. For a sixth-order polynomial expression in two independent variables, the number of coefficients to be determined is 28 . Hence the size of the non-singular sample set is 28 points. The software was then used to generate sample sets as multiples of 28 points, from 28 to 7 $\times 28$ points. Some of these are shown in figure 4 for a non-dimensionalised space. Note that the maximum number of sample points considered for fitting a surface through the data was 196, rather than the the much larger sample sets typically used in a conventional process.

\section{Generating D-Optimal sample data}

The most dense of the D-Optimal sampling schemes was run using an existing 5-hole probe, for which a prior dense conventional sample set of 1684 points in a regular grid had already been generated. As with the prior data for 
the 7-hole probe, the data acquired was fed into the Design of Experiments software for fitting as a polynomial response surface. Initial fits to each of the pressure responses for these points showed a standard deviation between the fitted surfaces and the sample points of typically 1.5 to $3 \%$ of dynamic pressure.

An RSM was obtained for $C_{P}$ for each of the five holes. The regression coefficient $R^{2}$ was in all cases greater than 0.999, and typical values of the standard deviation are shown in Table 1. The response surfaces were compared with both the sample set from which they were derived, and a densely sampled reference set measured using the same apparatus. These are shown as standard deviation of errors $\sigma$ for both sets of comparison points. Another characteristic was apparent: although the mean error against the sample set for which the data was generated was near zero, as expected, the mean error when compared against the reference sample set was finite, and different, for each pressure hole. Where the standard deviation of error against the reference set is comparable with, or smaller than, that from the sample set from which they were derived, the implication is that the results were subject to an offset of the mean error. This implies an effect in repeatability of pressure measurements between tests. Where the standard deviation against the reference sample set is higher than for the data from which they were derived, this implies a more complex interaction. This did highlight the importance of consistency of pressure measurements between runs and during longer runs, as the set of transducers in use at this stage were not temperaturecompensated, and thus subject to some small amount of time-dependent drift. However, the level of agreement for most of the pressure holes was considered an important proof-of-concept.

\begin{tabular}{|c|c|c|}
\hline$\sigma$ & $\begin{array}{c}\text { 196-point D-Optimal } \\
\text { sample set }\end{array}$ & 1684-point regular grid \\
\hline $\mathrm{C}_{\mathrm{P} 1}$ & $1.64 \%$ & $1.59 \%$ \\
\hline $\mathrm{C}_{\mathrm{P} 2}$ & $2.98 \%$ & $1.69 \%$ \\
\hline $\mathrm{C}_{\mathrm{P} 5}$ & $9.15 \%$ & $1.62 \%$ \\
\hline
\end{tabular}

Table 1, Quantitative parameters for response surface representations of probe outputs

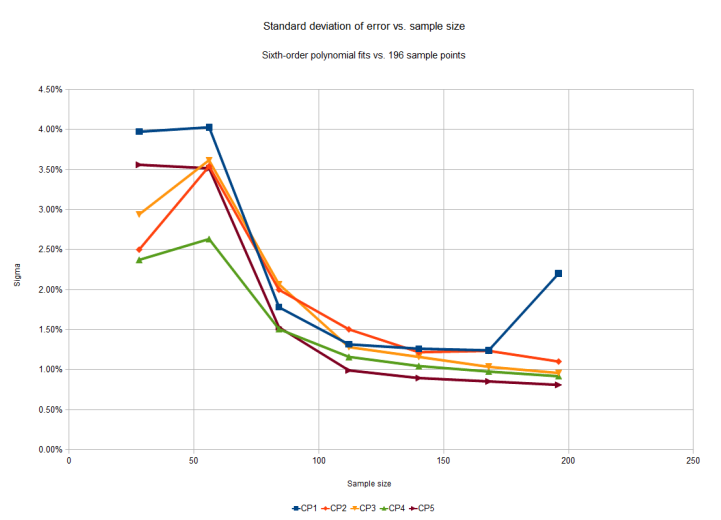

Fig. 5 Variation of standard deviation in pressure erro with sample size

In addition, the tunnel geometry was identified as a factor in the finite offset in static pressure measurements. The facility used has a constant section, and therefore a favourable pressure gradient along the length of the working section due to wall boundary layer growth. Extracting a static pressure measurement from a different streamwise location than that of the probe itself will therefore necessarily result in a finite offset. To examine this effect, an extra pitot-static probe was installed in the working section at the same downstream station as the probe itself, and the static pressure measurement used as a reference value for the five pressure transducers connected or to the probe. The upstream pitot-static system was still used to control the tunnel speed. This should not be as significant an issue for tunnels designed to have a zero longitudinal pressure gradient through the working section, but rig interference and associated blockage imply that static pressure measurement local to the probe is desirable.

Work is continuing, including the examination of how the parameters described above vary with the size of the sample set. Initial results for a five-hole probe are shown in figure 5. These show the variation of standard deviation of the errors between a series of response surfaces generated from successive sets of sample points of increasing size, when compared to a reference set corresponding to the largest of the sample sets. This set of tests have identified temperature-dependent drift as a primary factor in repeatability within and between tests, and further work will involve temperature-compensated pressure transducers.

In addition to updated results for five- and seven-hole probes, the final paper will include a treatment for multisensor hot wire probes, involving sampling in three-dimensional spaces. 


\section{Processing pressure measurements to produce attitude angles}

The nature of the variation in pressure between ports is such that a given value of pressure coefficient at one port will result in a contour or locus on the surface corresponding to the relationship

$$
C_{P i}=f_{i}(\theta, \psi)
$$

However, for a larger set of pressure holes, if these functions can be approximated adequately by a response surface, then a matrix could be defined relating the pressure coefficient vector $\mathbf{C}_{\mathbf{P}}$ to the attitude angle vector. As the nature of these functions is such that the intersection between two contours corresponding to different pressure holes is likely to have multiple values, pressures from one or more additional holes are needed to ensure that the attitude angles are determined uniquely. Determination of the attitude angles then becomes a matter of matrix inversion. There are two interesting corollaries to this approach:

1)Attitude angles can be determined uniquely with information from only three working holes, where at least four are required for the current technique.

2)The approach used to determine attitude angles can be adopted for an arbitrary number of pressure holes.

\section{Conclusion}

A formal approach to the optimization of the calibration of directional velocity probes has been developed by applying the theory of D-optimality. The multiple, independent calibrations required for a multi-hole pressure probe have been represented as response surfaces, and it has been shown that by selecting the appropriate points at which calibration data are collected, similar levels of measurement confidence may be obtained with an order of magnitude fewer calibration points.

\section{Acknowledgments}

This work has been funded by the U.K. Engineering and Physical Sciences Research Council under grant number EP/H030360/1. Mr. Jason Nunes is thanked for his contribution in the development and imaging of the seven-hole probe.

\section{References}

${ }^{1}$ Huffman, G. D., Rabe, D. C. and Poti, N. D. "Flow direction probes from a theoretical and experimental point of view." $J$. Phys. E.: Sci. Instrum. Vol. 13, No.7, 1980, pp. 751-760.

${ }^{2}$ Rediniotis, O. K. and Vijayagopal, R. "Miniature multihole pressure probes and their neural-network based calibration." AIAA Journal . Vol. 37, No. 6, 1999, pp. 666-674.

${ }^{3}$ Ericksen, A. L., Gallington, R. W., Rao, B. M. and Barankiewicz, W. S.,"Rapid calibration of seven-hole probes." NASA Technical Memorandum 107040, 1995.

${ }^{4}$ Wenger, C. W. and Devenport, W. J., "Seven-hole pressure probe calibration method utilizing look-up error tables." AIAA Journal, Vol. 37, No. 6, 1999 pp. 675-679.

${ }^{5}$ Zilliac, G. G. "Modeling, calibration and error analysis of 7-hole pressure probes." Exp. Fluids, Vol. 14, No. 1, 1993, pp. 104-120.

${ }^{6}$ Gameiro Silva, M. C., Pereira, C. A. C. and Cruz, J. M. S. "On the use of a linear interpolation method in the measurement procedure of a seven-hole pressure probe." Exp. Therm. \& Fluid Sci., Vol. 28, 2003, pp. 1-8.

${ }^{7}$ Sumner, D., "A comparison of data-reduction methods for a seven-hole probe." J. Fluids Eng., Vol. 124, 2002 , pp.523-527.

${ }^{8}$ Treaster, A. L. and Yocum, A. M. (1979) "The calibration and application of five-hole probes." ISA Trans. Vol. 18, No. 3, 1979, pp. 23-34.

${ }^{9}$ Pisasale, A. J. and Ahmed, N. A. "A novel method for extending the calibration range of five-hole probe for highly threedimensional flows." Flow Meas. \& Instr., Vol. 13, 2002, pp. 23-30.

${ }^{10}$ Zilliac, G., "Calibration of seven-hole pressure probes for use in fluid flows of large angularity." NASA Technical Memorandum 102200, 1989. 